\title{
Constant Riesz potentials on a circle in a plane with an application to polarization optimality problems
}

\author{
Nattapong Bosuwan ${ }^{\mathrm{a}, \mathrm{b}}$, Pornrat Ruengrot ${ }^{\mathrm{c}, *}$ \\ a Department of Mathematics, Faculty of Science, Mahidol University, Rama 6 Road, Ratchathewi District, \\ Bangkok 10400 Thailand \\ b Centre of Excellence in Mathematics, CHE, Si Ayutthaya Road, Bangkok 10400 Thailand \\ c Mahidol University International College, 999 Phutthamonthon 4 Road, Salaya, \\ Nakhonpathom 73170 Thailand
}

*Corresponding author, e-mail: pornrat.rue@mahidol.edu

Received 22 Jun 2017

Accepted 20 Aug 2017

\begin{abstract}
A characterization for a Riesz $s$-potential function of a multiset $\omega_{N}$ of $N$ points in $\mathbb{R}^{2}$ is given when $s=2-2 N$ and the potential function is constant on a circle in $\mathbb{R}^{2}$. The characterization allows us to partially prove a conjecture of Nikolov and Rafailov that if the potential function is constant on a circle $\Gamma$ then the points in $\omega_{N}$ should be equally spaced on a circle concentric to $\Gamma$. As an application of constant Riesz s-potential functions, we also find all maximal and minimal polarization constants and configurations of two concentric circles in $\mathbb{R}^{2}$ for certain values of $s$.
\end{abstract}

KEYWORDS: roots of unity, max-min and min-max problems

MSC2010: 52A40

\section{INTRODUCTION}

For a fixed multiset of $N$ points $\omega_{N}:=$ $\left\{x_{1}, x_{2}, \ldots, x_{N}\right\}$ in $\mathbb{R}^{2}$ and a given constant $s \in \mathbb{R}$, we define the Riesz potential function $U^{s}\left(\cdot ; \omega_{N}\right): \mathbb{R}^{2} \longrightarrow[0, \infty]$ as

$$
U^{s}\left(x ; \omega_{N}\right):=\sum_{j=1}^{N}\left|x-x_{j}\right|^{-s},
$$

where $x \in \mathbb{R}^{2}$ and $|\cdot|$ is the 2-dimensional Euclidean norm in $\mathbb{R}^{2}$. We call $U^{s}\left(\cdot ; \omega_{N}\right)$ a Riesz s-potential function of $\omega_{N}$. See Ref. 1 for more information on Riesz $s$-potential functions in a $d$-dimensional Euclidean space $\mathbb{R}^{d}$.

In this paper, we consider two problems concerning the Riesz $s$-potential functions $U^{s}\left(\cdot ; \omega_{N}\right)$. The first problem is to prove, in parts, Nikolov and Rafailov's conjecture about points in $\omega_{N}$ being equally spaced on some circle when a Riesz $s$ potential function is constant. The second problem is to solve polarization optimality problems when this Riesz $s$-potential function is constant.

Let $\omega_{N}$ be a fixed set of distinct equally spaced points on a circle $T \subseteq \mathbb{R}^{2}$ and $\Gamma$ be a circle concentric to $T$. In Ref. 2, Nikolov and Rafailov show in Theorem 1 that $U^{s}\left(x ; \omega_{N}\right)$ is constant as a function of $x$ on $\Gamma$ if and only if $s \in\{0,-2,-4, \ldots, 4-2 N, 2-$
$2 N\}$. They also show in Theorem 2 that this gives a characteristic property of distinct equally spaced points on a circle. More precisely, given a set $\omega_{N}$ of $N$ distinct points such that $U^{s}\left(x ; \omega_{N}\right)$ is constant on a circle $\Gamma$ for every $s \in\{-2,-4, \ldots, 2-2 N\}$ (the constant may depend on $s$ ), the points in $\omega_{N}$ are equally spaced on some circle concentric to $\Gamma$. In the same paper, it was conjectured (Conjecture 2) that only $s=2-2 N$ should be sufficient. We state the conjecture below.

Conjecture 1 Given a set of $N$ distinct points $\omega_{N}:=$ $\left\{x_{1}, x_{2}, \ldots, x_{N}\right\} \subseteq \mathbb{R}^{2}$ and a circle $\Gamma \subseteq \mathbb{R}^{2}$ such that

$$
U^{2-2 N}\left(x ; \omega_{N}\right)=\sum_{j=1}^{N}\left|x-x_{j}\right|^{2 N-2}
$$

is constant as a function of $x$ on $\Gamma$. Then $\omega_{N}$ forms a set of distinct equally spaced points on a circle concentric to $\Gamma$.

The conjecture was verified in the case $N=3$ (see Ref. 2, Proposition 2). In this paper, we prove Conjecture 1 in the following cases (after translating the centre of $\Gamma$ to the origin):

(i) when all points $x_{1}, x_{2}, \ldots, x_{N}$ have the same norm (Proposition 1); 
(ii) when $N=4$ and $x_{1}, x_{2}, x_{3}, x_{4}$ have an equal angle distribution (Proposition 2);

(iii) when $N$ is prime and $x_{1}, x_{2}, \ldots, x_{N}$ have an equal angle distribution and rational norms (Proposition 3).

The above results are based on a characterization of $\omega_{N}$ when $U^{2-2 N}\left(\cdot ; \omega_{N}\right)$ is constant on the unit circle (Theorem 1).

The next problems considered in this paper are polarization optimality problems. Let $\omega_{N}=$ $\left\{x_{1}, \ldots, x_{N}\right\}$ denote a configuration of $N$ (not necessarily distinct) points in $\mathbb{R}^{2}$. Denote by

$$
\mathbb{S}_{R}^{1}:=\left\{x \in \mathbb{R}^{2}:|x|=R\right\}
$$

the circle centred at the origin of radius $R$. When $R=1$, we simply use the notation $\mathbb{S}^{1}$. Given $s \in \mathbb{R}$, $R>0$, and $r>0$, we define polarization constants

$$
\begin{aligned}
& M_{N}^{s}\left(\mathbb{S}_{r}^{1} ; \mathbb{S}_{R}^{1}\right):=\max _{\substack{\omega_{N} \leq \mathbb{S}_{r}^{1} \\
\# \omega_{N}=N}} \min _{y \in \mathbb{S}_{R}^{1}} U^{s}\left(y ; \omega_{N}\right), \\
& M_{N}^{0}\left(\mathbb{S}_{r}^{1} ; \mathbb{S}_{R}^{1}\right):=N, \\
& m_{N}^{s}\left(\mathbb{S}_{r}^{1} ; \mathbb{S}_{R}^{1}\right):=\min _{\substack{\omega_{N} \leq \mathbb{S}_{r}^{1} \\
\# \omega_{N}=N}} \max _{y \in \mathbb{S}_{R}^{1}} U^{s}\left(y ; \omega_{N}\right), \\
& m_{N}^{0}\left(\mathbb{S}_{r}^{1} ; \mathbb{S}_{R}^{1}\right):=N,
\end{aligned}
$$

where $\# \omega_{N}$ denotes the cardinality of the multiset $\omega_{N}$. We will call $\omega_{N}$ a maximal (minimal) $N$-point Riesz s-polarization configuration of $\left(\mathbb{S}_{r}^{1} ; \mathbb{S}_{R}^{1}\right)$ if $\omega_{N}$ attains the maximum in (1) (minimum in (2)). We give a brief history of such polarization optimality problems below.

Farkas and Révész ${ }^{3}$ were the first to introduce two-plate polarization constants in a general sense. However, all previous results ${ }^{4-6}$ on polarization optimality problems related to Riesz potentials were considered for the case when $R=r=1$. The maximality of $N$ distinct equally spaced points on the unit circle for the maximal Riesz $s$-polarization problem of $\left(\mathbb{S}^{1} ; \mathbb{S}^{1}\right)$ in (1) was proved in Ref. 4 for $s=2$. Erdélyi and Saff ${ }^{1}$ established this for $s=$ 4. For arbitrary $s>0$, this result was proved in Ref. 5 where they also showed the minimality of $N$ distinct equally spaced points on the unit circle for the minimal Riesz $s$-polarization problem of $\left(\mathbb{S}^{1} ; \mathbb{S}^{1}\right)$ in (2) for $-1 \leqslant s<0$. Note that minimal $N$-point Riesz $s$-polarization problems of $\left(\mathbb{S}^{1} ; \mathbb{S}^{1}\right)$ when $s>0$ are not interesting because $m_{N}^{s}\left(\mathbb{S}^{1} ; \mathbb{S}^{1}\right)=\infty$ for all $s>0$.

Up to the present, there are no results on polarization optimality problems in (1) and (2) for
$R \neq r$. In this paper, we give a characterization of all maximal and minimal $N$-point Riesz $s$-polarization configurations of $\left(\mathbb{S}_{r}^{1} ; \mathbb{S}_{R}^{1}\right)$ when $s=-2,-4, \ldots, 2-$ $2 N$.

Although the asymptotic properties of polarization constants are not our main interest in this paper, it is worth mentioning the asymptotic types of behaviour of $M_{N}^{s}\left(\mathbb{S}^{1} ; \mathbb{S}^{1}\right)$ as $N \rightarrow \infty^{5}$ :

$$
M_{N}^{s}\left(\mathbb{S}^{1} ; \mathbb{S}^{1}\right) \sim \begin{cases}\frac{2 \zeta(s)}{(2 \pi)^{s}}\left(2^{s}-1\right) N^{s}, & s>1, \\ (1 / \pi) N \log N, & s=1, \\ \frac{2^{-s}}{\sqrt{\pi}} \frac{\Gamma\left(\frac{1-s}{2}\right)}{\Gamma\left(1-\frac{s}{2}\right)} N, & 0 \leqslant s<1,\end{cases}
$$

where $\zeta(s)$ denotes the classical Riemann zeta function and $a_{N} \sim b_{N}$ means that $\lim _{N \rightarrow \infty} a_{N} / b_{N}=1$. The reader is referred to Refs. 1, 7, 8 for asymptotic results of polarization constants and configurations of general subsets of $\mathbb{R}^{d}$ as $N \rightarrow \infty$ when $s>0$.

\section{CONSTANT RIESZ $s$-POTENTIAL FUNCTIONS}

The Euclidean space $\mathbb{R}^{2}$ and the complex space $\mathbb{C}$ over $\mathbb{R}$ have the same dimension and the same norm. However, the complex space $\mathbb{C}$ has a richer algebraic structure; for example, $\mathbb{C}$ is a field. Hence when we prove all theorems in this and the next section, any element $x \in \mathbb{R}^{2}$ will be replaced by $x \in$ $\mathbb{C}$, the 2-dimensional Euclidean norm $|\cdot|$ is replaced by the modulus in $\mathbb{C}$, and the notation $x y$ is adopted from the multiplication in $\mathbb{C}$ and the notation $x / y$ is adopted from the division in $\mathbb{C}$. We recall that the usual dot product in $\mathbb{C}$ is defined by

$$
\left(a_{1}+a_{2} \mathrm{i}\right) \cdot\left(b_{1}+b_{2} \mathrm{i}\right):=a_{1} b_{1}+a_{2} b_{2} .
$$

Now let $\omega_{N}:=\left\{x_{1}, x_{2}, \ldots, x_{N}\right\} \subseteq \mathbb{C}$ be a set of $N$ distinct points. In this section, we will assume that $U^{2-2 N}\left(x ; \omega_{N}\right)=\sum_{j=1}^{N}\left|x-x_{j}\right|^{2 N-2}$ is constant (as a function of $x$ ) on a circle $\Gamma \subset \mathbb{C}$ and prove that, under various conditions, the points $x_{1}, x_{2}, \ldots, x_{N}$ are equally spaced on some circle concentric to $\Gamma$. By translating and scaling the circle $\Gamma$, we can assume without loss of generality that $\Gamma$ is the unit circle $\mathbb{S}^{1}$. The following conjecture is equivalent to Conjecture 1 .

Conjecture 2 Given a set of $N$ distinct points $\omega_{N}:=$ $\left\{x_{1}, x_{2}, \ldots, x_{N}\right\} \subseteq \mathbb{C}$ such that

$$
U^{2-2 N}\left(x ; \omega_{N}\right)=\sum_{j=1}^{N}\left|x-x_{j}\right|^{2 N-2}
$$


is constant as a function of $x$ on $\mathbb{S}^{1}$, then $\omega_{N}$ forms a set of distinct equally spaced points on $\mathbb{S}_{R}^{1}$ for some $R$.

We begin with our main theorem which gives a characterization of $\omega_{N}$ when $U^{2-2 N}\left(\cdot ; \omega_{N}\right)$ is constant on the unit circle.

Theorem 1 Let $\omega_{N}=\left\{x_{1}, x_{2}, \ldots, x_{N}\right\} \subseteq \mathbb{C}$ be a set of $N$ distinct points. Then the function

$$
U^{2-2 N}\left(x ; \omega_{N}\right)=\sum_{j=1}^{N}\left|x-x_{j}\right|^{2 N-2}
$$

is constant on the circle $\mathbb{S}^{1}$ if and only if

$$
\begin{array}{r}
\sum_{j=1}^{N} \sum_{q=0}^{N-k-1}\left(\begin{array}{c}
N-1 \\
q
\end{array}\right)\left(\begin{array}{c}
N-1 \\
k+q
\end{array}\right)\left|x_{j}\right|^{2 N-2 k-2 q-2} x_{j}^{k} \\
=0, \quad \text { for all } \quad k=1, \ldots, N-1 .
\end{array}
$$

Note that (3) gives a system of $N-1$ equations in terms of elements in the set $\omega_{N}$. The proof of Theorem 1 requires a technical lemma which involves a lot of calculations, and so we will postpone it to the end of this section.

Example 1 Suppose $U^{2-2 N}\left(x ; \omega_{N}\right)$ is constant on $\mathbb{S}^{1}$. We list the systems of equations (3) that the $x_{j}$ must satisfy for small values of $N$ below.

(i) Let $N=3$. Then $x_{1}, x_{2}, x_{3}$ must satisfy

$$
\sum_{j=1}^{3} x_{j}^{2}=0, \quad \sum_{j=1}^{3}\left(1+\left|x_{j}\right|^{2}\right) x_{j}=0 .
$$

(ii) Let $N=4$. Then $x_{1}, x_{2}, x_{3}, x_{4}$ must satisfy

$$
\begin{gathered}
\sum_{j=1}^{4} x_{j}^{3}=0, \quad \sum_{j=1}^{4}\left(1+\left|x_{j}\right|^{2}\right) x_{j}^{2}=0, \\
\sum_{j=1}^{4}\left(1+3\left|x_{j}\right|^{2}+\left|x_{j}\right|^{4}\right) x_{j}=0 .
\end{gathered}
$$

(iii) Let $N=5$. Then $x_{1}, x_{2}, x_{3}, x_{4}, x_{5}$ must satisfy

$$
\begin{gathered}
\sum_{j=1}^{5} x_{j}^{4}=0, \quad \sum_{j=1}^{5}\left(1+\left|x_{j}\right|^{2}\right) x_{j}^{3}=0, \\
\sum_{j=1}^{5}\left(3+8\left|x_{j}\right|^{2}+3\left|x_{j}\right|^{4}\right) x_{j}^{2}=0, \\
\sum_{j=1}^{5}\left(1+5\left|x_{j}\right|^{2}+\left|x_{j}\right|^{4}\right)\left(1+\left|x_{j}\right|^{2}\right) x_{j}=0 .
\end{gathered}
$$

Using the characterization given in Theorem 1, we can verify Conjecture 2 in various cases. Our first result asserts that Conjecture 2 holds if the points $x_{1}, x_{2}, \ldots, x_{N}$ already lie on the same circle centred at the origin (i.e., they have the same norm).

Proposition 1 Let $\omega_{N}=\left\{x_{1}, x_{2}, \ldots, x_{N}\right\} \subseteq \mathbb{C}$ be a set of $N$ distinct non-zero points lying on some circle centred at the origin. If $U^{2-2 N}\left(\cdot ; \omega_{N}\right)$ is constant on $\mathbb{S}^{1}$, then $x_{1}, x_{2}, \ldots, x_{N}$ are equally spaced.

Proof: It suffices to show that $x_{1}, x_{2}, \ldots, x_{N}$ are the $N$ th roots of some complex number. Suppose $\left|x_{1}\right|=$ $\left|x_{2}\right|=\cdots=\left|x_{N}\right|=R$. From (3) we deduce that

$$
\sum_{j=1}^{N} x_{j}^{k}=0,
$$

for all $k=1,2, \ldots, N-1$. By Newton's identities,

$$
e_{k}\left(x_{1}, x_{2}, \ldots, x_{N}\right)=0, \quad k=1,2, \ldots, N-1,
$$

where the $e_{k}$ are elementary symmetric polynomials. Thus $x_{1}, x_{2}, \ldots, x_{N}$ are distinct roots of the polynomial

$$
\prod_{k=1}^{N}\left(X-x_{k}\right)=X^{N}-\mu
$$

for some $\mu \in \mathbb{C}$.

Now we will consider another special case. Instead of assuming that all points have the same norm, we will assume that they have an equal angle distribution around the origin. More precisely, let $\zeta=\mathrm{e}^{2 \pi \mathrm{i} / N}$ and, without loss of generality, we assume that

$$
x_{1}=r_{1} \zeta^{1}, x_{2}=r_{2} \zeta^{2}, \ldots, x_{N}=r_{N} \zeta^{N}
$$

for some positive real numbers $r_{1}, r_{2}, \ldots, r_{N}$. Our next result proves Conjecture 2 when $N=4$ and $x_{1}$, $x_{2}, x_{3}, x_{4}$ have an equal angle distribution.

Proposition 2 Let $x_{1}, x_{2}, x_{3}, x_{4}$ be as in (4). Suppose that

$$
U^{-6}\left(x ; \omega_{N}\right):=\sum_{j=1}^{N}\left|x-x_{j}\right|^{6}
$$

is constant as a function of $x$ on $\mathbb{S}^{1}$. Then $x_{1}, x_{2}, x_{3}$, $x_{4}$ are equally spaced on a circle centred at the origin.

Proof: By Proposition 1, it suffices to show that $\left|x_{1}\right|=\left|x_{2}\right|=\left|x_{3}\right|=\left|x_{4}\right|$. From Example 1, the points 
$x_{1}, x_{2}, x_{3}, x_{4}$ must satisfy

$$
\begin{aligned}
\sum_{j=1}^{4} x_{j}^{3} & =\sum_{j=1}^{4}\left(1+\left|x_{j}\right|^{2}\right) x_{j}^{2} \\
& =\sum_{j=1}^{4}\left(1+3\left|x_{j}\right|^{2}+\left|x_{j}\right|^{4}\right) x_{j}=0 .
\end{aligned}
$$

With $x_{j}=r_{j} \zeta^{j}$, the equation $\sum_{j=1}^{4} x_{j}^{3}=0$ becomes

$$
r_{1}^{3} \zeta^{3}+r_{2}^{3} \zeta^{2}+r_{3}^{3} \zeta+r_{4}^{3}=0
$$

Let $P(X)=r_{1}^{3} X^{3}+r_{2}^{3} X^{2}+r_{3}^{3} X+r_{4}^{3} \in \mathbb{R}[X]$. Since $\zeta=\mathrm{i}, \bar{\zeta}=-\mathrm{i}$ are roots of $P(X)$, we have

$$
P(X)=C\left(X^{2}+1\right)(X+b)=C\left(X^{3}+b X^{2}+X+b\right),
$$

for some non-zero $C \in \mathbb{R}$. Comparing the coefficients, we have $r_{1}=r_{3}, r_{2}=r_{4}$.

The equation $\sum_{j=1}^{4}\left(1+\left|x_{j}\right|^{2}\right) x_{j}^{2}=0$ becomes $\sum_{j=1}^{4}\left(1+r_{j}^{2}\right) r_{j}^{2} \zeta^{2 j}=0$. Expanding the sum and using $r_{1}=r_{3}, r_{2}=r_{4}$ we have

$$
2\left(1+r_{1}^{2}\right) r_{1}^{2} \zeta^{2}+2\left(1+r_{2}^{2}\right) r_{2}^{2}=0 .
$$

Since $\zeta^{2}=-1$ we obtain $\left(1+r_{1}^{2}\right) r_{1}^{2}=\left(1+r_{2}^{2}\right) r_{2}^{2}$. Let $t=r_{2} / r_{1}$ and $a=1 / r_{1}^{2}$. We have

$$
(a+1)=\left(a+t^{2}\right) t^{2} \Longrightarrow t^{4}+a t^{2}-(a+1)=0 .
$$

Thus

$$
t^{2}=\frac{-a \pm \sqrt{a^{2}+4 a+4}}{2}=\frac{-a \pm(a+2)}{2} .
$$

The only possible case is $t^{2}=\frac{1}{2}(-a+(a+2))=1$. Since $t>0$ we have $t=1$. Hence $r_{2}=r_{1}$. We have shown that $r_{1}=r_{2}=r_{3}=r_{4}$.

Actually, if we further assume that all norms are rational, then Conjecture 2 holds for all prime $N$.

Proposition 3 Let $N$ be a prime number. Let $x_{1}, x_{2}, \ldots, x_{N}$ be as in (4) where all $r_{j} \in \mathbb{Q}$. Suppose that $U^{2-2 N}\left(\cdot ; \omega_{N}\right)$ is constant on $\mathbb{S}^{1}$. Then $x_{1}, x_{2}, \ldots, x_{N}$ are equally spaced on a circle centred at the origin.

Proof: By Proposition 1, it suffices to show that $\left|x_{1}\right|=\left|x_{2}\right|=\cdots=\left|x_{N}\right|$. Applying the condition (3) with $k=N-1$ gives $\sum_{j=1}^{N} x_{j}^{N-1}=0$. Thus

$$
\sum_{j=1}^{N} r_{j}^{N-1} \zeta^{-j}=\sum_{j=1}^{N} r_{N-j}^{N-1} \zeta^{j}=0 .
$$

Let $A$ be a positive integer so that $A r_{N-j}^{N-1} \in \mathbb{Z}_{>0}$ for every $j$. Then $\sum_{j=1}^{N}\left(A r_{N-j}^{N-1}\right) \zeta^{k}=0$. This is a vanishing linear combination of $1, \zeta, \ldots, \zeta^{N-1}$ with positive-integer coefficients. Since the minimal polynomial of $\zeta$ is $1+X+\cdots+X^{N-1}$ ( $N$ is prime), this implies that all coefficients are equal. Thus $A r_{1}^{N-1}=A r_{2}^{N-1}=\cdots=A r_{N}^{N-1}$ and hence $r_{1}=r_{2}=$ $\cdots=r_{N}$.

\section{Proof of Theorem 1}

The following technical lemma is needed for the proofs of Theorem 1 and Theorem 2 .

Lemma 1 Let $N \in \mathbb{N}$ and $p \in\{1,2, \ldots, N-1\}$ be fixed. If $x_{j}:=\left|x_{j}\right| \cos t_{j}+\mathrm{i}\left|x_{j}\right| \sin t_{j}$ for all $j=$ $1,2, \ldots, N$, then for all $y:=\cos t+i \sin t \in \mathbb{S}^{1}$,

$$
\begin{aligned}
& \sum_{j=1}^{N}\left|y-x_{j}\right|^{2 p} \\
& =E_{0}+\sum_{k=1}^{p} \sum_{j=1}^{N}\left[E_{k, j} \cos k t_{j} \cos k t+E_{k, j} \sin k t_{j} \sin k t\right]
\end{aligned}
$$

where

$$
\begin{aligned}
E_{0} & =\sum_{j=1}^{N} \sum_{q=0}^{p}\left(\begin{array}{l}
p \\
q
\end{array}\right)^{2}\left|x_{j}\right|^{2 p-2 q}, \\
E_{k, j} & =(-1)^{k} 2 \sum_{q=0}^{p-k}\left(\begin{array}{l}
p \\
q
\end{array}\right)\left(\begin{array}{c}
p \\
k+q
\end{array}\right)\left|x_{j}\right|^{2 p-k-2 q} .
\end{aligned}
$$

Proof: Let $y:=\cos t+i \sin t \in \mathbb{S}^{1}$ and $x_{j}:=$ $\left|x_{j}\right| \cos t_{j}+\mathrm{i}\left|x_{j}\right| \sin t_{j}$ for all $j=1,2, \ldots, N$. A simple calculation shows that

$$
f_{j}(t):=\left|y-x_{j}\right|^{2 p}=\left(\left|x_{j}\right|^{2}+1-2\left|x_{j}\right| \cos \left(t-t_{j}\right)\right)^{p} .
$$

Since $A:=\left\{1, \cos \left(t-t_{j}\right), \ldots, \cos p\left(t-t_{j}\right)\right\}$ forms an orthogonal system with respect to the inner product

$$
\langle f, g\rangle:=\int_{0}^{2 \pi} f(t) g(t) \mathrm{d} t
$$

and $f_{j} \in \operatorname{span}(A)$, we have

$$
\begin{aligned}
f_{j}(t)=\sum_{k=0}^{p} E_{k, j} \cos k\left(t-t_{j}\right)=E_{0, j} \\
\quad+\sum_{k=1}^{p} E_{k, j}\left(\cos k t_{j} \cos k t+\sin k t_{j} \sin k t\right) .
\end{aligned}
$$


Now,

$$
\begin{aligned}
& \sum_{j=1}^{N}\left|y-x_{j}\right|^{2 p}=\sum_{j=1}^{N} f_{j}(t) \\
& =E_{0}+\sum_{k=1}^{p} \sum_{j=1}^{N}\left[E_{k, j} \cos k t_{j} \cos k t+E_{k, j} \sin k t_{j} \sin k t\right],
\end{aligned}
$$

where $E_{0}=\sum_{j=1}^{N} E_{0, j}$. By the orthogonality of the elements in the set $A$ and the calculation in Lemma 3 in the last section, we have

$$
E_{0}=\sum_{j=1}^{N} \frac{\left\langle f_{j}, 1\right\rangle}{2 \pi}=\sum_{j=1}^{N} \sum_{q=0}^{p}\left(\begin{array}{l}
p \\
q
\end{array}\right)^{2}\left|x_{j}\right|^{2 p-2 q}
$$

and

$$
\begin{aligned}
E_{k, j} & =\frac{\left\langle f_{j}, \cos k\left(t-t_{j}\right)\right\rangle}{\pi} \\
& =(-1)^{k} 2 \sum_{q=0}^{p-k}\left(\begin{array}{c}
p \\
q
\end{array}\right)\left(\begin{array}{c}
p \\
k+q
\end{array}\right)\left|x_{j}\right|^{2 p-k-2 q},
\end{aligned}
$$

for all $k \in\{1,2, \ldots, p\}$ and $j \in\{1,2, \ldots, N\}$.

Proof of Theorem 1: For each $j=1,2, \ldots, N$, set

$$
x_{j}:=\left|x_{j}\right| \cos t_{j}+\mathrm{i}\left|x_{j}\right| \sin t_{j} .
$$

$\Leftrightarrow)$ By our assumption, $f(y):=\sum_{j=1}^{N}\left|y-x_{j}\right|^{2 N-2}$ is constant on $\mathbb{S}^{1}$, say $f(y)=C$ on $\mathbb{S}^{1}$. Set

$$
y=\cos t+\mathrm{i} \sin t \in \mathbb{S}^{1} .
$$

By (5) for all $t \in[0,2 \pi]$,

$$
\begin{aligned}
C= & f(y)=E_{0} \\
& +\sum_{k=1}^{N-1} \sum_{j=1}^{N}\left[E_{k, j} \cos k t_{j} \cos k t+E_{k, j} \sin k t_{j} \sin k t\right] .
\end{aligned}
$$

Because the set $\{1, \cos t, \sin t, \ldots, \cos (N-$ 1) $t, \sin (N-1) t\}$ is linearly independent over $\mathbb{R}$, we deduce

$$
C-E_{0}=0
$$

and for all $k=1,2, \ldots, N-1$,

$$
\sum_{j=1}^{N} E_{k, j} \cos k t_{j}=0 \quad \text { and } \quad \sum_{j=1}^{N} E_{k, j} \sin k t_{j}=0 .
$$

Using the formulae of $E_{k, j}$ from Lemma 1, it follows from (7) that for all $k=1,2, \ldots, N-1$,

$$
\begin{gathered}
0=\sum_{j=1}^{N} E_{k, j}\left(\cos k t_{j}+\mathrm{i} \sin k t_{j}\right)=\sum_{j=1}^{N} \frac{E_{k, j}}{\left|x_{j}\right|} x_{j}^{k} \\
=(-1)^{k} 2 \sum_{j=1}^{N} \sum_{q=0}^{N-k-1}\left(\begin{array}{c}
N-1 \\
q
\end{array}\right)\left(\begin{array}{c}
N-1 \\
k+q
\end{array}\right) \\
\quad \times\left|x_{j}\right|^{2 N-2 k-2 q-2} x_{j}^{k},
\end{gathered}
$$

which implies (3).

$(\Leftarrow)$ Assume that (3) holds. Then we have (8) and (7). Combining (7) and the second identity in (6), we have for all $y \in \mathbb{S}^{1}$,

$$
\sum_{j=1}^{N}\left|y-x_{j}\right|^{2 N-2}=E_{0}
$$

which implies that $U^{2-2 N, h}\left(\cdot ; \omega_{N}\right)$ is constant on $\mathbb{S}^{1}$.

\section{AN APPLICATION TO POLARIZATION OPTIMALITY PROBLEMS}

We remind the reader that we will consider polarization optimality problems in the complex plane. A complete characterization of all maximal and minimal $N$-point Riesz $s$-polarization configurations of $\left(\mathbb{S}_{r}^{1} ; \mathbb{S}_{R}^{1}\right)$ when $s=-2,-4, \ldots, 2-2 N$ is given as follows.

Theorem 2 Let $N \in \mathbb{N}, p \in\{1,2, \ldots, N-1\}, R>$ $0, r>0$, and $\left\{x_{1}, x_{2}, \ldots, x_{N}\right\} \subseteq \mathbb{S}_{r}^{1}$. The following statements are equivalent:

(a) $\left\{x_{1}, x_{2}, \ldots, x_{N}\right\}$ is a maximal $N$-point Riesz $-2 p$ polarization configuration of $\left(\mathbb{S}_{r}^{1} ; \mathbb{S}_{R}^{1}\right)$;

(b) $\left\{x_{1}, x_{2}, \ldots, x_{N}\right\}$ is a minimal $N$-point Riesz $-2 p$ polarization configuration of $\left(\mathbb{S}_{r}^{1} ; \mathbb{S}_{R}^{1}\right)$;

(c) $\sum_{j=1}^{N} x_{j}=\sum_{j=1}^{N} x_{j}^{2}=\cdots=\sum_{j=1}^{N} x_{j}^{p}=0$.

Furthermore,

$$
\begin{aligned}
M_{N}^{-2 p}\left(\mathbb{S}_{r}^{1} ; \mathbb{S}_{R}^{1}\right)=m_{N}^{-2 p}\left(\mathbb{S}_{r}^{1} ; \mathbb{S}_{R}^{1}\right) \\
\quad=\frac{N}{2^{p}} \sum_{j=0}^{p}\left(\begin{array}{l}
p \\
j
\end{array}\right)^{2}(2 r R)^{2 j}\left(r^{2}+R^{2}+\left|r^{2}-R^{2}\right|\right)^{p-2 j} .
\end{aligned}
$$

Unlike the case when $R=r=1$ and $s>0$, optimal configurations for the cases in Theorem 2 may not be unique up to rotation. For example, when $p=$ 1 and $N=4$, our characterization of optimal configurations is $\sum_{j=1}^{4} x_{j}=0$. Hence there are infinitely many optimal configurations that are not rotations of one another. The proof of Theorem 2 relies on 
the fact that if $\omega_{N}$ is a configuration of $N$ distinct equally spaced points on $\mathbb{S}_{r}^{1}$, then for each $s=$ $-2,-4, \ldots, 2-2 N, U^{s}\left(\cdot, \omega_{N}\right)$ is constant on $\mathbb{S}_{R}^{1}$. This special property allows the problems to have more than one solution (up to rotation). Furthermore, our experimental study suggests that for the cases when $s \in \mathbb{R}^{2} \backslash\{0,-2,-4, \ldots, 2-2 N\}$, any maximal and minimal $N$-point Riesz $s$-polarization configuration of $\left(\mathbb{S}_{r}^{1} ; \mathbb{S}_{R}^{1}\right)$ is unique up to rotation, namely, it is a configuration of distinct equally spaced points on $\mathbb{S}_{r}^{1}$. We make the following conjecture.

Conjecture 3 Let $N \in \mathbb{N}, s \in \mathbb{R} \backslash\{0,-2,-4, \ldots, 2-$ $2 N\}, R>0, r>0$, and $\left\{x_{1}, x_{2}, \ldots, x_{N}\right\} \subseteq \mathbb{S}_{r}^{1}$. The following statements are equivalent:

(a) $\left\{x_{1}, x_{2}, \ldots, x_{N}\right\}$ is a maximal $N$-point Riesz $s$ polarization configuration of $\left(\mathbb{S}_{r}^{1} ; \mathbb{S}_{R}^{1}\right)$;

(b) $\left\{x_{1}, x_{2}, \ldots, x_{N}\right\}$ is a minimal $N$-point Riesz $s$ polarization configuration of $\left(\mathbb{S}_{r}^{1} ; \mathbb{S}_{R}^{1}\right)$;

(c) $\left\{x_{1}, x_{2}, \ldots, x_{N}\right\}$ is a configuration of distinct equally spaced points on $\mathbb{S}_{r}^{1}$.

\section{Proof of Theorem 2}

We need the following lemma.

Lemma 2 Let $N \in \mathbb{N}, p \in\{1,2, \ldots, N-1\}, R>0$, and $r>0$. Then any configuration of $N$ distinct equally spaced points on $\mathbb{S}_{r}^{1}$ is both a maximal and a minimal $N$-point Riesz -2p-polarization configuration of $\left(\mathbb{S}_{r}^{1} ; \mathbb{S}_{R}^{1}\right)$.

Proof: Let $\omega_{N}:=\left\{x_{1}, x_{2}, \ldots, x_{N}\right\}$ be a configuration of $N$ distinct equally spaced points on $\mathbb{S}_{r}^{1}$ and $p \in$ $\{1,2, \ldots, N-1\}$ be fixed. By Theorem 1 in Ref. 2, we know that $f(x):=\sum_{j=1}^{N}\left|x-x_{j}\right|^{2 p}$ is constant as a function of $x$ on $\mathbb{S}_{R}^{1}$, say $f(x)=C$ for all $x \in \mathbb{S}_{R}^{1}$. Thus

$$
\max _{x \in \mathbb{S}_{R}^{1}} \sum_{i=1}^{N}\left|x_{i}-x\right|^{2 p}=C=\min _{x \in \mathbb{S}_{R}^{1}} \sum_{i=1}^{N}\left|x_{i}-x\right|^{2 p} .
$$

Let $\left\{y_{1}, y_{2}, \ldots, y_{N}\right\}$ be any $N$-point configuration on $\mathbb{S}_{r}^{1}$. To show that $\omega_{N}$ is a minimal $N$-point Riesz $-2 p$-polarization configuration of $\left(\mathbb{S}_{r}^{1} ; \mathbb{S}_{R}^{1}\right)$, we will show that

$$
\max _{x \in \mathbb{S}_{R}^{1}} \sum_{i=1}^{N}\left|x_{i}-x\right|^{2 p} \leqslant \max _{x \in \mathbb{S}_{R}^{1}} \sum_{i=1}^{N}\left|y_{i}-x\right|^{2 p} .
$$

Consider

$$
\left|x_{j}-\frac{R}{y_{i} / r}\right|=\left|\frac{x_{j}}{y_{i}}\left(y_{i}-\frac{R}{x_{j} / r}\right)\right|=\left|y_{i}-\frac{R}{x_{j} / r}\right| \text {. }
$$

As $R /\left(y_{i} / r\right) \in \mathbb{S}_{R}^{1}$ for all $i$, we have

$$
\begin{aligned}
N C & =\sum_{i=1}^{N} f\left(\frac{R}{y_{i} / r}\right)=\sum_{i=1}^{N} \sum_{j=1}^{N}\left|x_{j}-\frac{R}{y_{i} / r}\right|^{2 p} \\
& =\sum_{j=1}^{N} \sum_{i=1}^{N}\left|y_{i}-\frac{R}{x_{j} / r}\right|^{2 p} .
\end{aligned}
$$

It follows from (12) that there is $j_{0} \in\{1,2, \ldots, N\}$ such that

$$
C \leqslant \sum_{i=1}^{N}\left|y_{i}-\frac{R}{x_{j_{0}} / r}\right|^{2 p} \leqslant \max _{x \in \mathbb{S}_{R}^{1}} \sum_{i=1}^{N}\left|y_{i}-x\right|^{2 p} .
$$

But $C=\max _{x \in \mathbb{S}_{R}^{1}} \sum_{i=1}^{N}\left|x_{i}-x\right|^{2 p}$ from (10). Hence we have (11) as required.

To show that $\omega_{N}$ is a maximal $N$-point Riesz $-2 p$-polarization configuration of $\left(\mathbb{S}_{r}^{1} ; \mathbb{S}_{R}^{1}\right)$, we will show that

$$
\min _{x \in \mathbb{S}_{R}^{1}} \sum_{i=1}^{N}\left|y_{i}-x\right|^{2 p} \leqslant \min _{x \in \mathbb{S}_{R}^{1}} \sum_{i=1}^{N}\left|x_{i}-x\right|^{2 p} .
$$

It follows from (12) that there is $j_{0}^{\prime} \in\{1,2, \ldots, N\}$ such that

$$
\min _{x \in \mathbb{S}_{R}^{1}} \sum_{i=1}^{N}\left|y_{i}-x\right|^{2 p} \leqslant \sum_{i=1}^{N}\left|y_{i}-\frac{R}{x_{j_{0}^{\prime}} / r}\right|^{2 p} \leqslant C .
$$

But $C=\min _{x \in \mathbb{S}_{R}^{1}} \sum_{i=1}^{N}\left|x_{i}-x\right|^{2 p}$ from (10). Hence we have (13) as required.

Proof of Theorem 2: Because the proof of (a) $\Leftrightarrow$ (c) is similar to the proof of (b) $\Leftrightarrow$ (c), we will show only (b) $\Leftrightarrow$ (c) and skip the proof of (a) $\Leftrightarrow$ (c). Without loss of generality, we can assume that $R=1$.

Let $N \in \mathbb{N}, p \in\{1,2, \ldots, N-1\}$, and $r>0$ be fixed and $\left\{x_{1}, x_{2}, \ldots, x_{N}\right\}$ be any configuration in $\mathbb{S}_{r}^{1}$. We recall from Lemma 1 that for $x_{j}:=$ $r \cos t_{j}+\mathrm{i} r \sin t_{j}$ for all $j=1,2, \ldots, N$ and for all $y:=\cos t+\mathrm{i} \sin t \in \mathbb{S}^{1}$,

$$
\begin{aligned}
\sum_{j=1}^{N} \mid y & -\left.x_{j}\right|^{2 p}=E_{0} \\
& +\sum_{k=1}^{p} \sum_{j=1}^{N}\left[E_{k, j} \cos k t_{j} \cos k t+E_{k, j} \sin k t_{j} \sin k t\right],
\end{aligned}
$$

$$
=E_{0}+\sum_{k=1}^{p} \sum_{j=1}^{N}\left[\frac{E_{k, j}}{r^{k}}\left(y^{k} \cdot x_{j}^{k}\right)\right],
$$


where

$$
\begin{aligned}
E_{0} & =\sum_{j=1}^{N} \sum_{q=0}^{p}\left(\begin{array}{l}
p \\
q
\end{array}\right)^{2} r^{2 p-2 q}, \\
\frac{E_{k, j}}{r^{k}} & =(-1)^{k} 2 \sum_{q=0}^{p-k}\left(\begin{array}{l}
p \\
q
\end{array}\right)\left(\begin{array}{c}
p \\
k+q
\end{array}\right) r^{2 p-2 k-2 q} .
\end{aligned}
$$

Notice that the constant $E_{0}$ does not depend on a configuration on $\mathbb{S}_{r}^{1}$ and the constants $E_{k, j} / r^{k}$ do not depend on a configuration on $\mathbb{S}_{r}^{1}$ and $j$. For convenience for all configurations $\left\{x_{1}, x_{2}, \ldots, x_{N}\right\} \subseteq$ $\mathbb{S}_{r}^{1}$, we set

$$
E_{k}:=\frac{E_{k, j}}{r^{k}}, \quad \text { for all } k=1,2, \ldots, p .
$$

First of all, we will show that

$$
m_{N}^{-2 p}\left(\mathbb{S}_{r}^{1} ; \mathbb{S}^{1}\right)=E_{0} .
$$

Let $\omega_{N}^{\prime}:=\left\{x_{1}^{\prime}, x_{2}^{\prime}, \ldots, x_{N}^{\prime}\right\}$ be a configuration of distinct equally spaced points on $\mathbb{S}_{r}^{1}$. Using (15), we have for all $y \in \mathbb{S}^{1}$,

$$
\begin{array}{r}
\sum_{j=1}^{N}\left|y-x_{j}^{\prime}\right|^{2 p}=E_{0}+\sum_{k=1}^{p} \sum_{j=1}^{N} E_{k}\left(y^{k} \cdot\left(x_{j}^{\prime}\right)^{k}\right) \\
=E_{0}+\sum_{k=1}^{p} E_{k}\left(y^{k} \cdot \sum_{j=1}^{N}\left(x_{j}^{\prime}\right)^{k}\right)=E_{0}
\end{array}
$$

where the last equality follows from the fact that $\sum_{j=1}^{N}\left(x_{j}^{\prime}\right)^{k}=0$ for all $k=1,2, \ldots, p$. Since $\omega_{N}^{\prime}$ is a minimal $N$-point Riesz $-2 p$-polarization configuration of $\left(\mathbb{S}_{r}^{1} ; \mathbb{S}^{1}\right)$ (by Lemma 2 ), we obtain

$$
m_{N}^{-2 p}\left(\mathbb{S}_{r}^{1} ; \mathbb{S}^{1}\right)=\max _{y \in \mathbb{S}^{1}} U^{-2 p}\left(y ; \omega_{N}^{\prime}\right)=E_{0} .
$$

We now prove $(\mathrm{c}) \Rightarrow(\mathrm{b})$. Assume that $\omega_{N}=$ $\left\{x_{1}, x_{2}, \ldots, x_{N}\right\} \subseteq \mathbb{S}_{r}^{1}$ such that $\sum_{j=1}^{N} x_{j}^{k}=0$ for all $k=1,2, \ldots, p$. Applying the same argument as in (18), we have for all $y \in \mathbb{S}^{1}$,

$$
U^{-2 p}\left(y ; \omega_{N}\right)=E_{0}+\sum_{k=1}^{p} E_{k}\left(y^{k} \cdot \sum_{j=1}^{N} x_{j}^{k}\right)=E_{0},
$$

which implies that $\omega_{N}$ is a minimal $N$-point Riesz $-2 p$-polarization configuration of $\left(\mathbb{S}_{r}^{1} ; \mathbb{S}^{1}\right)$.

Next, we show (b) $\Rightarrow$ (c). Assume that $\omega_{N}=$ $\left\{x_{1}, x_{2}, \ldots, x_{N}\right\}$ is a minimal $N$-point Riesz $-2 p$ polarization configuration of $\left(\mathbb{S}_{r}^{1} ; \mathbb{S}^{1}\right)$. Then for all $y \in \mathbb{S}^{1}$,

$$
U^{-2 p}\left(y ; \omega_{N}\right)=\sum_{j=1}^{N}\left|y-x_{j}\right|^{2 p} \leqslant m_{N}^{-2 p}\left(\mathbb{S}_{r}^{1} ; \mathbb{S}^{1}\right)=E_{0} .
$$

Then, by (14) and (17) for all $t \in[0,2 \pi]$,

$E_{0} \geqslant U^{-2 p}\left(y ; \omega_{N}\right)=E_{0}+\sum_{k=1}^{p}(\mathscr{C} \cos k t+\mathscr{S} \sin k t)$.

where $\mathscr{C}=\sum_{j=1}^{N} E_{k} \cos k t_{j}$ and $\mathscr{S}=\sum_{j=1}^{N} E_{k} \sin k t_{j}$. Thus for all $t \in[0,2 \pi]$,

$$
0 \geqslant \sum_{k=1}^{p}(\mathscr{C} \cos k t+\mathscr{S} \sin k t) .
$$

Hence for all $t \in[0,2 \pi]$,

$$
\sum_{k=1}^{p}(\mathscr{C} \cos k t+\mathscr{S} \sin k t)=0 .
$$

Because $\{\cos t, \sin t, \cos 2 t, \sin 2 t, \ldots, \cos p t, \sin p t\}$ is a linearly independent set over $\mathbb{R}$ for all $k=$ $1,2, \ldots, p$,

$$
\sum_{j=1}^{N} E_{k} \cos k t_{j}=\sum_{j=1}^{N} E_{k} \sin k t_{j}=0 .
$$

Since for all $k=1,2, \ldots, p, E_{k} \neq 0((16))$,

$$
\sum_{j=1}^{N} \cos k t_{j}=\sum_{j=1}^{N} \sin k t_{j}=0, \quad k=1,2, \ldots, p,
$$

which implies that $\sum_{j=1}^{N} x_{j}^{k}=\sum_{j=1}^{N} r^{k}\left(\cos k t_{j}+\right.$ $\left.i \sin k t_{j}\right)=0$ for all $k=1,2, \ldots, p$.

To compute $M_{N}^{-2 p}\left(\mathbb{S}_{r}^{1} ; \mathbb{S}_{R}^{1}\right)$ and $m_{N}^{-2 p}\left(\mathbb{S}_{r}^{1} ; \mathbb{S}_{R}^{1}\right)$ in (9), we can use a similar argument in Lemma 1 by replacing $y=R \cos t+\mathrm{i} R \sin t$ and $f_{j}(t)=\left|y-x_{j}\right|^{2 p}=$ $\left(r^{2}+R^{2}-2 R r \cos \left(t-t_{j}\right)\right)^{p}$. Applying the calculations as in Lemma 4, it is not difficult to check that if $\omega_{N}$ is a configuration of $N$ distinct equally spaced points on $\mathbb{S}_{r}^{1}$, then for all $y \in \mathbb{S}_{R}^{1}$,

$$
\begin{aligned}
& U^{-2 p}\left(y ; \omega_{N}\right) \\
& =\frac{N}{2^{p}} \sum_{j=0}^{p}\left(\begin{array}{c}
p \\
j
\end{array}\right)^{2}(2 r R)^{2 j}\left(r^{2}+R^{2}+\left|r^{2}-R^{2}\right|\right)^{p-2 j} .
\end{aligned}
$$

\section{COMPUTATIONS OF INTEGRALS}

We collect our computations of all integrals in this section.

Lemma 3 Let $p \in \mathbb{N}, k \in\{0,1, \ldots, p\}$, and $z \in \mathbb{C}$. Then

$$
\begin{aligned}
& \int_{0}^{2 \pi}\left(z^{2}+1-2 z \cos t\right)^{p} \cos k t \mathrm{~d} t \\
& \quad=(-1)^{k} 2 \pi \sum_{q=0}^{p-k}\left(\begin{array}{l}
p \\
q
\end{array}\right)\left(\begin{array}{c}
p \\
k+q
\end{array}\right) z^{2 p-k-2 q} .
\end{aligned}
$$


Proof: Let $p \in \mathbb{N}$ and $k \in\{0,1, \ldots, p\}$. First, we prove the equality (19) for $z \in \mathbb{R}$. Let $z \in \mathbb{R}$. Then, for $\zeta=\mathrm{e}^{\mathrm{i} t}$,

$$
\begin{aligned}
\int_{0}^{2 \pi} & \left(z^{2}+1-2 z \cos t\right)^{p} \cos k t \mathrm{~d} t \\
= & \int_{0}^{2 \pi}\left(z^{2}+1-z\left(\mathrm{e}^{\mathrm{i} t}+\mathrm{e}^{-\mathrm{i} t}\right)\right)^{p} \mathrm{e}^{\mathrm{i} k t} \mathrm{~d} t \\
= & \int_{0}^{2 \pi}\left(z-\mathrm{e}^{\mathrm{i} t}\right)^{p}\left(z-\mathrm{e}^{-\mathrm{i} t}\right)^{p} \mathrm{e}^{\mathrm{i} k t} \mathrm{~d} t \\
= & \frac{1}{\mathrm{i}} \int_{\mathbb{S}^{1}}(z-\zeta)^{p}(z-1 / \zeta)^{p} \zeta^{k-1} \mathrm{~d} \zeta \\
= & 2 \pi \operatorname{Res}\left(\frac{(z-\zeta)^{p}(z \zeta-1)^{p}}{\zeta \zeta} \zeta=0\right) \\
= & (-1)^{k} 2 \pi \sum_{q=0}^{p-k}\left(\begin{array}{c}
p \\
q
\end{array}\right)\left(\begin{array}{c}
p \\
k+q
\end{array}\right) z^{2 p-k-2 q},
\end{aligned}
$$

where the first equality follows from the fact that the last expression is a real number. Notice that the left-hand side and the right-hand side of (19) are polynomials as functions of $z$. Thus both functions are analytic on $\mathbb{C}$ and we have (19) for all $z \in \mathbb{C}$.

Lemma 4 Let $p \in \mathbb{N}$ and $k \in\{0,1, \ldots, p\}$. For $a, b \in$ $\mathbb{C}$,

$$
\begin{aligned}
\int_{0}^{2 \pi}(a-b \cos t)^{p} \cos k t d t \\
\quad=\frac{(-1)^{k} \pi}{2^{p-1}} \sum_{q=0}^{p-k}\left(\begin{array}{l}
p \\
q
\end{array}\right)\left(\begin{array}{c}
p \\
k+q
\end{array}\right) C_{a, b, p, q, k},
\end{aligned}
$$

where $C_{a, b, p, q, k}=b^{2 q+k}\left(a \pm \sqrt{a^{2}-b^{2}}\right)^{p-k-2 q}$ and the square root function in (20) can be selected to be both branches of the complex square root function.

Proof: Clearly, if $b=0$, then the equation in (20) is $0=0$. Assume that $b \in \mathbb{C} \backslash\{0\}$ and $a \in \mathbb{C}$. To reduce (20) to (19), we consider

$$
(\lambda a-\lambda b \cos t)^{p}
$$

where $\lambda$ is chosen to satisfy the equations

$$
2 z=b \lambda, \quad z^{2}+1=a \lambda,
$$

for some $z \in \mathbb{C}$. From the above equations,

$$
z=\frac{a \pm \sqrt{a^{2}-b^{2}}}{b}
$$

and

$$
\lambda=\frac{2 z}{b}=\frac{2 a \pm 2 \sqrt{a^{2}-b^{2}}}{b^{2}} .
$$

Furthermore, $\lambda \neq 0$ because if $\lambda=0$, then $z=0$ which implies that $b=0$. Hence by Lemma 3,

$$
\begin{aligned}
\int_{0}^{2 \pi} & (a-b \cos t)^{p} \cos k t \mathrm{~d} t \\
= & \frac{1}{\lambda p} \int_{0}^{2 \pi}(\lambda a-\lambda b \cos t)^{p} \cos k t \mathrm{~d} t \\
= & \frac{1}{\lambda p} \int_{0}^{2 \pi}\left(z^{2}+1-2 z \cos t\right)^{p} \cos k t \mathrm{~d} t \\
= & \frac{(-1)^{k} \pi}{2^{p-1}} \sum_{q=0}^{p-k}\left(\begin{array}{c}
p \\
q
\end{array}\right)\left(\begin{array}{c}
p \\
k+q
\end{array}\right) C_{a, b, p, q, k} .
\end{aligned}
$$

Acknowledgements: This study was supported by the Strengthen Research Grant for New Lecturers from the Thailand Research Fund and the Office of the Higher Education Commission (MRG6080133) and Faculty of Science, Mahidol University. Furthermore, the authors are grateful to Dr Chatchawan Panraksa for his helpful comments on the topic of this manuscript.

\section{REFERENCES}

1. Erdélyi T, Saff EB (2013) Riesz polarization inequalities in higher dimensions. J Approx Theor 171, 128-47.

2. Nikolov N, Rafailov R (2013) On extremums of sums of powered distances to a finite set of points. Geom Dedicata 167, 69-89.

3. Farkas B, Révész Sz Gy (2006) Potential theoretic approach to rendezvous numbers. Monatsh Math 148, 309-31.

4. Ambrus G, Ball K, Erdélyi T (2013) Chebyshev constants on the unit ball. Bull Lond Math Soc 45, 236-48.

5. Hardin DP, Kendall AP, Saff EB (2013) Polarization optimality of equally spaced points on the circle for discrete potentials. Discrete Comput Geom 50, 236-43.

6. Nikolov N, Rafailov R (2011) On the sum of powered distances to certain sets of points on the circle. Pac $J$ Math 253, 157-68.

7. Borodachov SV, Bosuwan N (2014) Asymptotics of discrete Riesz $d$-polarization on subsets of $d$-dimensional manifolds. Potential Anal 41, 35-49.

8. Borodachov SV, Hardin DP, Reznikov A, Saff EB (to appear) Optimal discrete measures for Riesz potentials. Trans Amer Math Soc. 